- regulations and the growth of renewable energy sources, especially wind power.

But first, the regulations must withstand inevitable industry lawsuits intended to weaken or overturn them. "The power-plant rule is really pivotal to Obama's legacy, and it is going to face tough legal scrutiny," says David Victor, director of the Laboratory on International Law and Regulation at the University of California, San Diego. If it succeeds, he adds, "it could reverse the position of the United States internationally".

Although Obama was unable to secure climate legislation during his first term, his administration did achieve gains in the wake of the recession. It secured billions of dollars in stimulus funding for clean energy, efficiency measures and green infrastructure, as well as establishing significant new standards for vehicle emissions and fuel economy. That, combined with the economic slowdown and the shift away from coal in the electricity sector, means that US greenhouse-gas emissions have already decreased by around $10 \%$ since 2005 .

In theory, the administration still has both the time and the means to reduce emissions

enough for the United States to meet its international commitments, says Kennedy. "They will have to take very serious action, but the tools that they have available to them should allow them to do it."

Republicans have vowed to challenge Obama at every turn. They started the current session with a debate on legislation to approve the con-

\section{"The power-}

plant rule is

really pivotal to

Obama's legacy, and it is going to face tough legal scrutiny." troversial Keystone XL pipeline, which would carry crude oil from the tar sands of Alberta, Canada, to refineries on the US Gulf Coast. The House of Representatives quickly passed a bill to approve the pipeline, but partisan disagreements have delayed a Senate vote. Obama has promised to veto the legislation.

Although the pipeline would have a small effect on global greenhouse-gas emissions, it has become a symbolic issue for both sides of the climate debate. On 21 January, Senate Democrats used the Keystone fight to confront Republicans on their views about climate by putting to a vote declarations about human involvement in global warming (see Nature http://doi.org/zpx; 2015). Fifteen Republicans supported an amendment to the Keystone bill stating that climate change is affected by human activity, and five voted for an amendment stating that climate change is "significantly" affected by humans.

Although neither amendment passed, those votes are a sign that Republicans are feeling pressure and may warm to certain climate solutions in future, says Bob Inglis, a Republican former member of the House who heads the Energy and Enterprise Initiative, a think tank that advocates for conservative environmental solutions at George Mason University in Fairfax, Virginia. Although Inglis understands why Obama has chosen to sidestep Congress and address climate change with regulations, he says that the president still has a potential opportunity to secure his environmental legacy by striking a grand legislative bargain with his Republican opposition.

"Obama is in a box," says Inglis, "but he could get out of that box if he were a little bit bolder." - SEE EDITORIALP. 527

\title{
Philae hunt hangs in the balance
}

\section{Rosetta mission would have to sacrifice other science to search for comet lander.}

\section{BY ELIZABETH GIBNEY}

$\mathrm{T}$ The lost space probe Philae, which made history after it landed on a comet last November, is posing a dilemma for scientists at the European Space Agency (ESA). They have what is probably their last chance to change the path of Philae's parent craft, Rosetta, to hunt for the lander, which went missing shortly after it touched down on comet 67P/Churyumov-Gerasimenko. But the shift would also mean sacrificing some of Rosetta's long-planned science observations.

The agonizing choice comes as the mission team published its first batch of papers from observations made after Rosetta entered into orbit around 67P last August - reporting a varied landscape and hinting at the comet's origins.

Philae has been silent since its batteries ran out just days after its bumpy landing on 12 November. On the basis of images of its initial bounces and data from radio instruments, Philae's position has been narrowed down to a 20 -metre by 200 -metre strip. But efforts to find the 1-metre-wide lander in highresolution pictures taken by Rosetta from a distance of about 20 kilometres have so far failed.

\section{NEARLY WEIGHTLESS}

The resulting effect of gravitational potential and centrifugal forces, mapped on coment $67 \mathrm{P} /$ Churyumov-Gerasimenko, is revealed to be greatest on the lobes and weaker in the neck region.

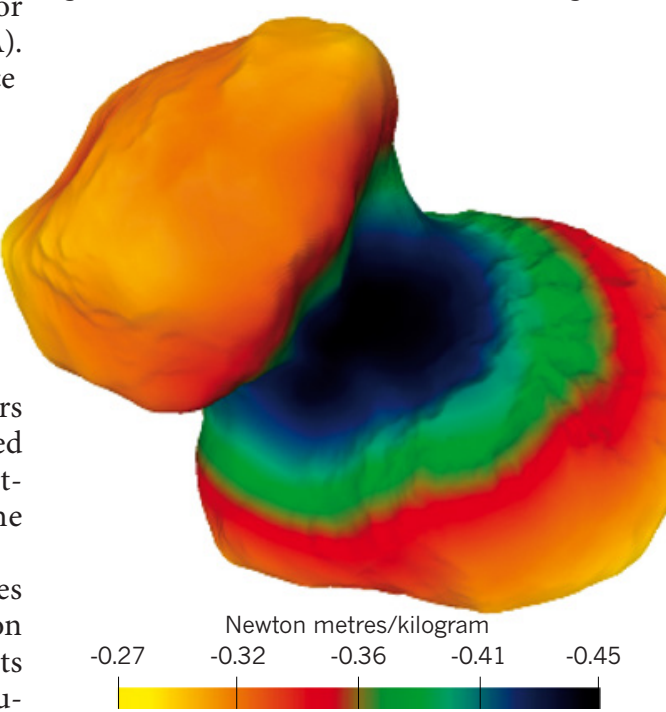

Project scientists are debating whether to send Rosetta, which is still orbiting the comet, down to an altitude of 6 kilometres, over the patch where Philae is thought to be. It would be the closest that the craft has ever been to 67P. But Rosetta has limited fuel. Any attempt to look for Philae would mean scrapping a different flyby, which would offer the chance to image the comet in a shadow-free shot that should reveal fine details about the surface structure and composition.

As the comet approaches the Sun, growing cometary surface activity in the form of jets of gas and dust also makes it increasingly risky for Rosetta to approach. If Rosetta is to stick to the original flyby plan, scheduled for 14 February, the craft will not come as close to 67P until 2016, says the mission's flight director Andrea Accomazzo, after the comet has swung around the Sun and headed back out to space.

\section{SURFACE SEARCH}

There are scientific benefits to pinpointing Philae's location, says Wlodek Kofman, principal investigator on Rosetta's CONSERT (Comet Nucleus Sounding Experiment by Radiowave Transmission) experiment, which is designed to send radio waves between the

\section{ONATURE.COM}

For the best images from Rosetta's data haul, see:

go.nature.com/rrihsj 
parent craft and Philae to study the comet's interior. Not knowing the lander's exact location makes it much harder to process the data that scientists have already received and to generate accurate results, he says. Spotting the lander would also help to determine its exact location and angle, and to predict how likely it is to come back to life in the coming months as the comet nears the Sun and its solar panels begin to receive more light, Kofman says.

The decision is not an easy one, says Holger Sierks, who is principal investigator on Rosetta's OSIRIS (Optical, Spectroscopic, and Infrared Remote Imaging System) instrument.

The mission has already produced a haul of results, which were published in a series of papers in Science on 22 January (see Nature http://doi.org/zpz; 2015). Using data from OSIRIS and the Radio Science Investigation instrument, Sierks and his collaborators calculated the gravity on the rubber-duck-shaped comet and created a map (see 'Nearly weightless') that also takes into account the centrifugal force caused by the comet's rotation (H. Sierks et al. Science http://doi.org/zp2; 2015). The resulting force is greatest on top of the lobes, but it is about six times weaker in the neck region, where dust can lift off more easily. The team also used the data to calculate the comet's density, finding that the body is relatively fluffy and porous - with a density of around half that of water, giving clues to its structure and strength.

The researchers described three-metrewide pebble-like features that are found all over the comet, which they nicknamed "goosebumps". Sierks says that the shapes could hint at the size of the grains of dust and ice that first clumped together in the early Solar System before forming larger bodies. "The hypothesis is these might be the building blocks of comets," he says.

In another of the papers, OSIRIS data enabled Sierks and his collaborators to classify the geography of the comet's surface on the basis of terrain types. These include fractures, possible impact craters and an array of dunes and ripples that may have been formed by gas travelling around the surface, like wind shaping sand in a desert (N. Thomas et al. Science http:// doi.org/zp3; 2015).

The final word on whether to send Rosetta to look for Philae rests with ESA. Kofman says that an informal vote among Rosetta scientists came down narrowly on the side of doing it. As Nature went to press, the agency was thought to be leaning towards sticking to its original agenda, because looking for Philae would mean too much upheaval for the mission.

If ESA decides against a mission shift to hunt for Philae, the team could still get lucky: it may find clues as to the lander's whereabouts either in existing images or in new shots taken from flybys between $20 \mathrm{~km}$ and $50 \mathrm{~km}$ away in the coming months. Finding Philae in these kind of flybys is not impossible, says Accomazzo, "but it would be sheer luck".

PSYCHOLOGY

\section{Clash over 'smart unconscious'}

\section{Report examining decisions made while distracted adds to controversy about the power of the unconscious.}

\section{BY ALISON ABBOTT}

$\mathrm{I}$ f you have to make a complex decision, will you do a better job if you absorb yourself in, say, a crossword puzzle instead of ruminating about your options? The idea that unconscious thought is sometimes more powerful than conscious thought is attractive, and echoes ideas popularized by books such as writer Malcolm Gladwell's best-selling Blink.

But within the scientific community, 'unconscious-thought advantage' (UTA) has been controversial. Now Dutch psychologists have carried out the most rigorous study yet of UTA - and find no evidence for it.

Their conclusion, published this week in Judgement and Decision Making, is based on a large experiment that they designed to provide the best chance of capturing the effect should it exist, along with a sophisticated statistical analysis of previously published data ${ }^{1}$.

The report adds to broader concerns about the quality of psychology studies and to an ongoing controversy about the extent to which unconscious thought in general can influence behaviour. "The bigger debate is about how clever our unconscious is," says cognitive psychologist David Shanks of University College London. "This carefully constructed paper makes a great contribution." Shanks published a review last year that questioned research claiming that various unconscious influences, including UTA, affect decision making ${ }^{2}$.

A typical study probing UTA asks subjects to make a complex decision, such as choosing a car or a computer, after either mulling over a list of the object's attributes or viewing the list quickly and then engaging in a distracting activity such as a word puzzle. However, such studies have drawn different conclusions, with about half of those published so far reporting a UTA effect and the other half finding none.

Proponents of the theory claim that the effect is exquisitely sensitive to experimental variations, and often attribute the negative results to the fact that many research groups varied elements of the set-up, such as the choice of puzzle used for the distraction ${ }^{3}$.
Critics say that the positive results came from having too few participants in the experiments.

Psychologists Mark Nieuwenstein and Hedderik van Rijn at the University of Groningen in the Netherlands set out with their colleagues to determine which explanation was correct.

They asked 399 participants - around ten times more than the typical (median) sample sizes in other studies - to choose between either 4 cars or 4 apartments on the basis of 12 desirable or undesirable features. They incorporated the full list of conditions that UTA proponents had reported as yielding the strongest effect, such as the exact type of puzzle used as a distraction. They found that the distracted group
"How we make decisions, and how we might make them better, has practical and intellectual importance." was no more likely than the deliberating group to choose the most desirable item.

The scientists then reanalysed 60 of the 81 experiments described in the 32 UTA papers published before April 2014. For this 'meta-analysis', they excluded experiments that had insufficient data for analysis or that deviated from conditions that are reported as likely to elicit UTA (only one of these experiments had claimed a UTA effect). They also included the results of their own study. When they applied a rigorous statistical meta-analysis, they found no significant UTA effect.

"Psychologists have historically prided themselves on their command of statistics," says psychologist Jonathan Baron at the University of Pennsylvania in Philadelphia, the editor of Judgement and Decision Making. But this study shows that many in the past were poorly designed. He adds: "If UTA is out there, it can't be captured in experiments designed in the lab."

Psychologist Ap Dijksterhuis at Radboud University in Nijmegen, the Netherlands, who first described ${ }^{4}$ unconscious-thought theory, which predicts UTA, in 2004, says: "It is certainly true that psychology has 